\title{
Comparison of response and acute toxicities of concurrent chemoradiation with weekly cisplatin vs. paclitaxel/ carboplatin in locally advanced squamous cell carcinoma of head \& neck: Protocol of a short-term phase III trial
}

\author{
Md Shuayb ${ }^{1 *}$ and Md Shah Jalalur Rahman Shahi ${ }^{2}$ \\ ${ }^{1}$ Square Oncology \& Radiotherapy Centre, Square Hospitals Ltd., Dhaka, Bangladesh \\ ${ }^{2}$ Ahsania Mission Cancer \& General Hospital, Dhaka, Bangladesh
}

\begin{abstract}
Concurrent chemoradiation therapy (CCRT) has become the treatment of choice for locally advanced squamous cell carcinoma of head \& neck (SCCHN). Multiple chemoradiation treatment paradigms are existent, but unfortunately the optimum timing, dosing and choice of systemic agents are controversial. Even though the current most widely accepted standard chemotherapy for radiation sensitization remains cisplatin, the toxicities are significant. Carboplatin possesses radiationsensitizing properties as a result of its ability for repairing sublethal damage, binding thiols and inducing chromosomal aberrations. Compared to cisplatin, it is less nephrotoxic, neurotoxic and ototoxic. Paclitaxel is also a potent radiosensitizer, as demonstrated in preclinical and clinical trials, due to its effect of inducing cell cycle arrest in G2/M phase. The combining of carboplatin and paclitaxel with radiation has demonstrated promising clinical activity in respect of efficacy and improved tolerability against newly diagnosed, recurrent and metastatic SCCHN. This study intends to explore the advantage of multiagent CCRT with taxane over single agent cisplatin for patients with locally advanced, non-metastatic SCCHN. Patients with stage-III \& IVA/B SCCHN will be randomly enrolled to receive following treatments: CDDP arm-Cisplatin $\left(30 \mathrm{mg} / \mathrm{m}^{2}\right)$ weekly plus radiotherapy (RT) (66-70 Gy, $1.8-2 \mathrm{~Gy} /$ fraction); PC arm-weekly paclitaxel (40mg/m²) and carboplatin (AUC-2) plus RT (66-70 Gy, 1.8-2 Gy/ fraction). Patients will be followed-up weekly and six weeks after completion of treatment, and treatment response will be evaluated with RECIST criteria. Toxicities will be assessed by RTOG Acute Radiation Morbidity Criteria. Chi-squared test, Fisher's exact test and t-test will be employed to compare between two treatment arms.
\end{abstract}

Ethics and dissemination: The study is registered at German Clinical Trials Register (DRKS00012877) and "National Institute of Cancer Research \& Hospital Ethics Committee", Bangladesh (Ethical Clearance Certificate Reference: NICRH/Ethics/2015/185). Results will be disseminated through peer-reviewed international journals and major international conferences.

Trial registration number: DRKS00012877. http://www.drks.de/DRKS00012877

\begin{abstract}
Abbreviations: AUC: Area Under the Curve; CCRT: Concurrent Chemoradiation Therapy; CT: Computed Tomography; ECOG: Eastern Cooperative Oncology Group; Gy: Gray; LRC: Loco Regional Control; OS: Overall Survival; PFS: Progression Free Survival; RECIST: Response Evaluation Criteria for Solid Tumor; RT: Radiotherapy; RTOG: Radiation Therapy Oncology Group; SCCHN: Squamous Cell Carcinoma of Head and Neck; SPSS: Statistical Package for Social Sciences
\end{abstract}

\section{Article Summary}

\section{Article focus}

1. Our hypothesis is that taxane containing regimen is superior to cisplatin only regimen in combination with radiation therapy for locally advanced SCCHN.

Key messages

1. For SCCHN, different chemotherapy regimens can be used with Radiation therapy where Cisplatin and Paclitaxel/Carboplatin both are acceptable options.

2. This randomized prospective trial will answer whether the integration of taxane into CCRT regimen will benefit patients of this group.

\section{Strengths and limitations of this study}

1. This is a short-term intervention and follow-up allowing the assessment of treatment response and acute toxicity.

2. Long-term outcome like late toxicity and survival will not be assessed in this short period of study.

3. The study will be conducted with all types of SCCHN in general.

\section{Background}

For many tumors, concurrent chemoradiotherapy (CCRT) has a central role in the treatment of locoregional disease. The use of CCRT in head \& neck cancer is important because locoregional control is pivotal here. This is the fifth most common cancer worldwide, with an estimated annual global incidence of 529,451 cases [1]. 90-95\% head

${ }^{\star}$ Correspondence to: Md Shuayb, Square Oncology \& Radiotherapy Centre, Square Hospitals Ltd., 18/F, West Panthapath, Dhaka 1205, Bangladesh, Tel: +8801553139179; E-mail: m.shuayb@yahoo.co.uk; drshuayb@squarehospital.com

Received: April 27, 2018; Accepted: May 23, 2018; Published: May 28, 2018 
Shuayb (2018) Comparison of response and acute toxicities of concurrent chemoradiation with weekly cisplatin vs. paclitaxel/carboplatin in locally advanced squamous cell carcinoma of head \& neck: Protocol of a short-term phase III trial

\& neck cancer diagnosis are squamous cell carcinomas (SCCHN) [2]; hence it is this type of disease should be focused on.

For practical purposes, SCCHN is divided into three clinical stages: early, locoregional/locally advanced, and metastatic or recurrent. Treatment approaches can vary depending on the disease stage. Most patients in the less developed regions in the world are diagnosed at locally advanced stage. In the past, survival at 5 years for locally advanced disease was reported to be only 40\% [3] (10-30\% for patients with stage IVA and IVB tumors) [4], and locoregional failure was the predominant cause of recurrence. More than $50 \%$ of patients who die from SCCHN have locoregional disease as the only site of failure, and almost $90 \%$ of patients with distant failure also have persistent locoregional disease [5-7]. Therefore, it is clear that the efficacy of any curative approach is measured by its ability to achieve local control.

Historically, locally advanced tumors were treated with surgery (with or without adjuvant radiotherapy) or radiotherapy alone. Only a minority of patients with locally advanced disease can undergo adequate surgical resection, and the outcomes were poor with respect to survival and organ preservation [4]. Radiotherapy alone is not sufficient to successfully treat most SCCHN at intermediate or advanced stages.

Currently, three different treatment options are available for locally advanced head \& neck squamous cell cancers. All of these three treatment options are multimodality treatment approaches sequential or concurrent. The first and foremost approach involves definitive surgery; which is followed by adjuvant CCRT or RT alone, which ensures accurate pathologic staging and precise identification and documentation of high-risk features that guides the adjuvant treatment. Although this approach may fail to preserve valuable organ like larynx, it ensures treatment guided by histopathological diagnosis $[8,9]$.

The alternate approach includes definitive concurrent chemoradiotherapy (CCRT) with salvage surgery as an optional backup treatment plan. This treatment approach lacks the pathologic information, a setback which is balanced by improved organ preservation. This benefit is already established for laryngeal cancer but is increasingly recognized for other anatomic locations; however, this approach remains controversial for oral cavity tumors [2].

The third approach uses of neoadjuvant chemotherapy followed by definitive surgery or radiation with curative intent. Major advantages include rapid reduction in tumor bulk in responders and the potential to decrease the risk of distant failure. Oftentimes response to induction predicts responsiveness to following definitive chemoradiotherapy. However, this can result in prolonged treatment time and additional chemotherapy-related toxic effects from systemic doses. This sequential approach also increases the total cost of treatment. This approach remains controversial for valid reasons, and is currently under investigation in several large, multicenter, randomized trials in order to determine significant benefit over CCRT $[2,10]$.

Nonetheless, sensitizing effects are not tumor specific and affect adjacent normal tissues within the radiation field [2]. CCRT trials have consistently reported an increased incidence of acute grade 3 and 4 toxic effects, with mucositis and dermatitis being the most prominent [2]. This creates concern about chronic toxic effects, including consequential late effects, which evolve from persistent severe acute toxic effects. Interestingly, multiple studies have confirmed that, compared with radiation alone, the long-term side effects of CCRT, such as on swallowing function or speech, are not increased [3,11-13]. Owing to the prominent incidence of acute toxic effects, treatment should preferentially be performed at experienced centers, in which improved overall outcomes are observed [14].

Various chemotherapeutic agents including cisplatin, 5-fluorouracil, bleomycin, hydroxyurea, paclitaxel, docetaxel, carboplatin, mitomycin C, methotrexate, pemetrexed [15] and tirapazamine, and targeted therapies including cetuximab, gefitinib [16] and bevacizumab [17] have been tested as single agents in combination with radiotherapy [2,18-22]. Further research has suggested that combination chemotherapy regimens offer the potential to improve response rates further and possibly improve survival [23-25]. Multiagent based CCRT are investigated with 5-FU/cisplatin, paclitaxel/cisplatin, cetuximab/ cisplatin, tirapazamine/cisplatin, paclitaxel/carboplatin, 5-FU/ carboplatin and 5-FU/hydroxyurea [2].

Cisplatin is a potent radiosensitizer and the drug most commonly used for CCRT in head \& neck cancer. A meta-analysis examining various chemoradiotherapy regimens indicated that platinum containing regimens might provide a survival advantage compared with non-cisplatin containing regimens [14]. Currently, the most widely used standard regimen is $100 \mathrm{mg} / \mathrm{m}^{2}$ cisplatin every 3 weeks, combined with $\sim 70$ Gy radiation delivered in 1.8-2.0 Gy daily fractions. This regimen causes severe toxic effects, such as nephro-, oto- and neurotoxic effects, nausea and vomiting, as well as severe mucositis, which make the treatment suitable only for patients with normal creatinine clearance and a good performance status. Furthermore, locoregional failure rates are 35-65\%, depending on tumor location, stage, and resectability [11-13]. To limit toxic effects, alternative administration schedules are also being used, but equivalent efficacy has not been established. For example, with once-weekly $30 \mathrm{mg} / \mathrm{m}^{2}$ cisplatin regimen, no nephrotoxic effects were reported, but mucositis and neutropenia were prominent [26].

Carboplatin is a second-generation platinum agent that is structurally and functionally similar to cisplatin $[27,28]$. However, the radiosensitizing properties of carboplatin are not as well established as those of cisplatin [2]. One Head \& neck cancer trial comparing cisplatin with carboplatin demonstrated similar efficacy and survival [29]. Carboplatin is frequently used in combination with other radiosensitizers, such as paclitaxel. The side effects of carboplatin are more favorable than those seen with cisplatin-based CCRT because few nephrotoxic or neurotoxic effects arise [2,18]. Nevertheless, hematologic toxic effects are slightly increased, and grade $3 / 4$ toxic effects are seen in $40 \%$ of patients $[2,18]$. Single-agent carboplatin based CCRT has a favorable toxicity profile for patients with SCCHN, and this regimen is usually used in combination with paclitaxel.

Taxanes are potent radiosensitizers, and studies have examined single-agent paclitaxel based CCRT in locally advanced SCCHN $[30,31]$. Paclitaxel is tolerated with radiotherapy when administered at weekly doses of up to $40 \mathrm{mg} / \mathrm{m}^{2}$ in SCCHN [32]. Mucositis and leukopenia are reported, but these toxic effects are generally predictable and manageable [2].

The radiation-sensitizing properties of both paclitaxel and carboplatin, as well as their documented activity in SCCHN have, therefore, motivated us to design a phase III trial. This project aims to assess short-term toxicity and efficacy of low-dose, multiagent chemotherapy with paclitaxel/carboplatin and single agent cisplatin given weekly in concurrently with, daily external beam radiotherapy.

Hypothesis of the trial: Our hypothesis is that taxane containing regimen is superior to cisplatin only regimen in combination with radiation therapy for locally advanced SCCHN. 


\section{Methods/Design}

\section{Study population}

The diagnosis of locally advanced stage III, IVA or IVB SCCHN will be confirmed by a radiation oncologist prior to the initiation of the treatment. Histopathological confirmation will be done for all patients. Physical examination, panendoscopy, CT scan of neck \& face as well as chest radiograph and ultrasound of abdomen will be done to determine the extent of disease and to exclude distant metastases. The patients will be staged according to the tumour-node-metastasis (TNM) classification. Pretherapy dental evaluation will be required prior to the start of chemoradiation. Patients will be selected randomly from National Institute of Cancer Research \& Hospital, who will meet the eligibility criteria of the study.

\section{Inclusion criteria}

- SCCHN proved by histopathology

- AJCC stage-III, IVA and IVB

- Age: 18 to 75

- Eastern Cooperative Oncology Group (ECOG) performance status $\leq 2$

- Biochemical tests values: WBC $\geq 3.5 \times 10^{9} / \mathrm{L}$, Neutrophils $\geq 1.5 \times 10^{9} / \mathrm{L}$, Platelets $\geq 100 \times 10^{9} / \mathrm{L}$, haemoglobin $\geq 9 \mathrm{gm} / \mathrm{dl}$, Aspartate aminotransferase (AST), alanine aminotransferase (ALT), and total bilirubin $\leq 1.5 \times$ the upper limit of normal range, Creatinine concentration $\leq 120 \mathrm{umol} / \mathrm{L}$, and creatinine clearance $\geq 50 \mathrm{ml} / \mathrm{min}$

- No serious diseases of important organs

- Written informed consent signed prior to enrollment.

\section{Exclusion criteria}

- Prior chemotherapy or head \& neck irradiation

- Pregnant or lactating woman

- Serious diseases of important organs

- Other malignancies

- Active uncontrolled infection

- Joined in other clinical trial

\section{Operational definition}

There will be two arms. In each arm 50 patients will be enrolled randomly.

CDDP Arm: Concurrent chemoradiotherapy regimens with CDDP (cisplatin $30 \mathrm{mg} / \mathrm{m}^{2}$ weekly with premedications and adequate hydration; radiotherapy- 6600-7000 cGy in single daily 1.8-2 Gy/ fraction, 5 days a week on linear accelerator with 4 or $6 \mathrm{MV}$ photon beams).

PC Arm: Concurrent chemoradiotherapy regimens with PC (paclitaxel $40 \mathrm{mg} / \mathrm{m}^{2}$ over 1 hour followed by carboplatin AUC-2 over 30 min with premedications, weekly; radiotherapy- 6600-7000 cGy in single daily 1.8-2 Gy/fraction, 5 days a week on linear accelerator with 4 or $6 \mathrm{MV}$ photon beams).

\section{Primary outcome measure}

Primary endpoint of this study is treatment response. Treatment response will be measured 6 weeks after completion of treatment according to the Response Evaluation Criteria in Solid Tumours (RECIST), version 1.1 as complete response (CR), partial response (PR), progressive disease (PD), stable disease (SD) using the data of panendoscopic evaluation and CT scans of neck \& face obtained 6 weeks after therapy. Pathologic confirmation will be required for patients suspected to have clinical evidence of residual disease at the primary site 6 weeks after therapy.

\section{Secondary outcome measure}

Secondary endpoint of this study is treatment related acute toxicities. Acute toxicities will be reported weekly during treatment and 6 weeks after completion of treatment. Acute toxicities of the two regimens will be evaluated by determining the frequency of severe $(\geq$ grade 3 ) toxicities based on RTOG Acute Radiation Morbidity Criteria using the information of history and physical examinations, ECOG performance status, and blood tests like $\mathrm{CBC}$, electrolyte, creatinine, ALT etc.

\section{Randomization}

All the locally advanced head \& neck cancer patients from National Institute of Cancer Research \& Hospital, Dhaka, Bangladesh will be assigned serial numbers. Then random number table will be used to choose each single patient until desired sample size is allocated to each group.

\section{Blinding}

The study is double blinded. Both patients and treating physician will be blinded about treatment groups.

\section{Sample size calculation}

Based on the data from recent relevant trials, standardized difference was calculated Using Altman's normogram and verified by Quick formula.

\section{Ethical implications}

- Institutional permission to collect data was obtained before conducting the study.

- Participants will be volunteered.

- All patients will be included in the study after informing about the nature of the study. They will be explained about the aim, objective, procedure, risk and benefit of the procedure in easily understandable language.

- Informed written consents will be obtained from the patients.

- All patients will be coded by a serial number which can be referenced to the chart number only.

- All participants will be free to take part or refuse to be a part of the study.

- The study will not interfere with patient management or deal with moral or social issue.

- The study protocol was submitted to the ethical review committee of the institution and an ethical clearance certificate was obtained.

- The study protocol was submitted to WHO Primary Registry German Clinical Trials Register for trial registration and was officially registered. 
Shuayb (2018) Comparison of response and acute toxicities of concurrent chemoradiation with weekly cisplatin vs. paclitaxel/carboplatin in locally advanced squamous cell carcinoma of head \& neck: Protocol of a short-term phase III trial

\section{Statistical analysis}

Data will be expressed by adding error bars which will show $+/$ standard deviation. Differences in patient demographics between CDDP and PC treated patients will be analyzed with chi-squared tests or two-sided student's t-tests. Fisher's exact test or chi-squared test will be used to compare treatment arms with respect to toxicity rates and response. Statistical co-relation will be done by SPSS (Statistical Package for the Social Sciences) software. A value of $\mathrm{P}<0.05$ will be considered statistically significant with confidence interval of $95 \%$.

\section{Discussion}

Head \& neck cancer is a worldwide health problem. More than $90 \%$ of these cancers are of squamous cell histology [33]. The majority of patients present with locoregionally advanced disease [33] and are managed with combined modality approaches. Newer treatment strategies that incorporate a combination of systemic agents and radiation (CCRT) are being widely investigated in this setting with the goal of improving both locoregional and distant disease control.

Recent trials have shown that CCRT offers a significant advantage over surgery followed by radiotherapy [18] or radiotherapy alone [3438], and induction chemotherapy followed by CCRT has shown no demonstrable benefit over CCRT for patients with locally advanced SCCHN [10]. Despite a substantial number of clinical trials performed to justify the safety and efficacy of several CCRT regimens, important questions on the optimal treatment paradigm remain. While the most commonly used chemotherapeutic agent in combination with RT has been cisplatin, multi-agent regimen, paclitaxel/carboplatin, has evolved with encouraging result in terms of efficacy and tolerability. $[2,18,33]$.

Patients with head \& neck cancer, most of the time, present with non-metastatic state. Advanced stage without distant disease (locally advanced) is curable in $50 \%$ cases with the introduction of an aggressive treatment protocol [2]. Concurrent chemoradiation is, although, based on the most robust evidence, unfortunately, is hampered by severe toxicity, and patients must be selected carefully before treatment [39]. The experience of the staff (physicians and nurses), and in particular its familiarity with toxicity management, as well as the structural facilities, play an important role in the final outcome [39]. Poor performance status and coexisting illness are other causes which restrain concomitant chemoradiation. Thus the optimal CCRT regimens remain questioned related to safety, efficacy and cost effectiveness. Hence a lot of patients experience locoregional or distant progression of their diseases and die within 5 years of diagnosis. Continued development with participation in clinical trials is, therefore, an immense need for further improvement on the treatment of patients with locally advanced SCCHN.

To the best of our knowledge, no clinical trial has hitherto been conducted in Bangladesh to directly compare the CCRT protocols over another. For this reason we are taking an attempt to analyze the safety and efficacy of the two effective CCRT regimens, weekly cisplatin and weekly paclitaxel/carboplatin. This project may provide a precise idea regarding the ideal CCRT protocol for locally advanced SCCHN patients. On completion of this short-term study, a large study may be embarked on to determine long-term outcome including long-term toxicity, overall survival, and progression free survival.

\section{Declarations}

\section{Acknowledgements}

We thank the authority of National Institute of Cancer Research \& Hospital, Dhaka, Bangladesh for approving the study to be conducted at this institution.

\section{Funding}

This research received no specific grant from any funding agency in the public, commercial or not-for-profit sectors.

\section{Authors' contributions}

Md Shuayb is the principal investigator (PI) of this trial, and developed the study concept, designed the protocol and wrote the manuscript. Md Shah Jalalur Rahman Shahi reviewed the manuscript. Md Shuayb is responsible for acquisition of clinical information from the patients, follow up of the patients, data interpretation and statistical analysis. Both authors read and approved the final manuscript.

\section{Consent for publication}

Informed written consent will be obtained from all patients for publication of this study and any accompanying images (with appropriate anonymity). A copy of the written consent is available for review by the Editor-in-Chief of this journal.

\section{Ethics approval and consent to participate}

The study protocol was approved by the Chairman of "National Institute of Cancer Research \& Hospital Ethics Committee" on $22^{\text {nd }}$ July, 2015 and an Ethical Clearance Certificate was obtained (Reference number of the Ethical Clearance Certificate: NICRH/Ethics/2015/185). Later the study protocol was submitted to German Clinical Trials Register for trial registration and was officially registered on 16th August, 2017 (Trial Registration number: DRKS00012877). URL of registry: http://www.drks.de/DRKS00012877 Enrollment of the first participant to the trial is yet to begin. All patients will be included in the study after informing about the aim, objective, procedure, risk and benefit of the procedure in easily understandable language and informed written consents for the participation will be obtained individually from all. Participants' privacy and confidentiality will be maintained strictly.

\section{Availability of data}

The study results will be published through peer-reviewed international journals and major international conferences.

\section{Competing interests}

The authors declare that they have no competing interests.

\section{References}

1. Ferlay J, Soerjomataram I, Ervik M, Dikshit R, Eser S, Mathers C, et al. (2013) GLOBOCAN 2012 v1.0, Cancer Incidence and Mortality Worldwide: IARC Cancer Base No. 11 [Internet]. Lyon, France: International Agency for Research on Cancer; Available from: http://globocan.iarc.fr

2. Seiwert TY, Salama JK, Vokes EE (2007) Chemoradiotherapy paradigm in Head \& neck cancer. Nature Clinical Practice Oncology 4: 156-171. [Crossref]

3. Laramore GE, Scott CB, al-Sarraf M, Haselow RE, Ervin TJ, et al. (1992) Adjuvant chemotherapy for resectable squamous cell carcinomas of the head and neck: report on Intergroup Study 0034. Int J Radiat Oncol Biol Phys 23: 705-713. [Crossref]

4. Vokes EE, Weichselbaum RR, Lippman SM, Hong WK (1993) Head and neck cancer N Engl J Med 328: 184-194. [Crossref]

5. Bloom C, Just N, Remy H, Black M, Rossignol M (1998) Laryngeal cancer: is computed tomography a valuable imaging technique? A retrospective analysis. Can Assoc Radiol J 49: 370-377. [Crossref]

6. Haughey BH, Arfken CL, Gates GA, Harvey J (1992) Meta-analysis of second malignant tumors in head and neck cancer: the case for an endoscopic screening protocol. Ann Otol Rhinol Laryngol 101: 105-112. [Crossref] 
Shuayb (2018) Comparison of response and acute toxicities of concurrent chemoradiation with weekly cisplatin vs. paclitaxel/carboplatin in locally advanced squamous cell carcinoma of head \& neck: Protocol of a short-term phase III trial

7. Guardiola E, Pivot X, Dassonville O, Poissonnet G, Marcy PY, et al. (2004) Is routine triple endoscopy for head and neck carcinoma patients necessary in light of a negative chest computed tomography scan? Cancer 101: 2028-2033. [Crossref]

8. Forastiere AA, Goepfert H, Maor M, Pajak TF, et al. (2003) Concurrent chemotherapy and radiotherapy for organ preservation in advanced laryngeal cancer. $N$ Engl J Med 349: 2091-2098. [Crossref]

9. Wolf GT, Fisher SG, Hong WK, Hillman R, Spaulding M, et al. (1991) Induction chemotherapy plus radiation compared with surgery plus radiation in patients with advanced laryngeal cancer. $N$ Engl J Med 324: 1685-1690. [Crossref]

10. Haddad R, O'Neill A, Rabinowits G, Tishler R, Khuri F, et al. (2013) Induction chemotherapy followed by concurrent chemoradiotherapy (sequential chemoradiotherapy) versus concurrent chemoradiotherapy alone in locally advanced head \& neck cancer (PARADIGM): a randomized phase 3 trial. Lancet Oncol 14: 257 264. [Crossref]

11. Bernier J, Domenge C, Ozsahin M, Matuszewska K, Lefèbvre JL, et al. (2004) Postoperative irradiation with or without concomitant chemotherapy for locally advanced head and neck cancer. N Engl J Med 350: 1945-1952. [Crossref]

12. Cooper JS, Pajak TF, Forastiere AA, Jacobs J, Campbell BH, et al. (2004) Postoperative concurrent radiotherapy and chemotherapy for high-risk squamous-cell carcinoma of the head and neck. N Engl J Med 350: 1937-1944. [Crossref]

13. Bachaud JM, Cohen-Jonathan E, Alzieu C, David JM, Serrano E, et al. (1996) Combined postoperative radiotherapy and weekly cisplatin infusion for locally advanced head and neck carcinoma: final report of a randomized trial. Int J Radiat Oncol Biol Phys 36: 999-1004. [Crossref]

14. Benasso M, Bonelli L, Numico G, Corvo R, Sanguineti G, et al. (1997) Treatment with cisplatin and fluorouracil alternating with radiation favourably affects prognosis of inoperable squamous cell carcinoma of the head and neck: results of a multivariate analysis on 273 patients. Ann Oncol 8: 773-779. [Crossref]

15. Seiwert TY, Connell PP, Mauer AM, Hoffman PC, George CM, et al. (2007) A phase I study of pemetrexed, carboplatin, and concurrent radiotherapy in patients with locally advanced or metastatic non-small cell lung or esophageal cancer. Clin Cancer Res 13: 515-522. [Crossref]

16. Cohen EEW, Haraf DJ, Stenson KM, Blair E, Brockstein BE, et al. (2005) Integration of gefitinib (G), into a concurrent chemoradiation (CRT) regimen followed by $G$ adjuvant therapy in patients with locally advanced head and neck cancer (HNC) - a phase II trial. J Clin Oncol 23: 5506.

17. Seiwert TY, Haraf DJ, Cohen EE, Stenson K, Witt ME, et al. (2008) Phase I study of bevacizumab added to fluorouracil- and hydroxyurea-based concomitan chemoradiotherapy for poor-prognosis head and neck cancer. J Clin Oncol 26: 17321741. [Crossref]

18. Suntharalingam M, Haas ML, Conley BA, Egorin MJ, Levy S, et al. (2000) The use of carboplatin and paclitaxel with daily radiotherapy in patients with locally advanced squamous cell carcinomas of the head and neck. Int. J. Radiation Oncology. Biol Phys 47: 49-56. [Crossref]

19. Bourhis J, Eschwege F (1996) Radiotherapy-chemotherapy combinations in head and neck squamous cell carcinoma: Overview of randomized trials. Anticancer Res 16 : 2397-2402. [Crossref]

20. Eisenberger M, Hornedo J, Silva H, Donehower R, Spaulding M, et al. (1986) Carboplatin (NSC-241-240): an active platinum analog for the treatment of squamouscell carcinoma of the head and neck. J Clin Oncol 4: 1506-1509. [Crossref]

21. Rowinsky EK, Donehower RC (1995) Paclitaxel (taxol) N Engl J Med 332: 1004-1014. [Crossref]

22. Vokes EE, Weichselbaum RR, Mick R, J McEvilly, Haraf DJ, et al. (1992) Favorable long term survival following induction chemotherapy with cisplatin, fluorouracil, and leucovorin and concomitant chemoradiotherapy for locally advanced head and neck cancer. J Natl Cancer Inst 84: 877- 882. [Crossref]
23. Haraf DJ, Vokes EE, Panje WR, Weichselbaum RR (1991) Survival and analysis of failure following hydroxyurea, 5-fluorouracil and concomitant radiation therapy in poor prognosis head and neck cancer. Am J Clin Oncol 14: 419-426. [Crossref]

24. Brizel DM, Albers ME, Fisher SR, Scher RL, Richtsmeier WJ, et al. (1998) Hyperfractionated irradiation with or without concurrent chemotherapy for locally locally advanced head and neck cancer. $N$ Engl J Med 338: 1798 -1804. [Crossref]

25. Vokes EE, Awan AM, Weichselbaum RR (1991) Radiotherapy with concomitan chemotherapy for head and neck cancer. Hematol Oncol Clin North Am 5: 753-767. [Crossref]

26. Cmelak AJ, Li S, Goldwasser MA, Murphy B, Cannon M, et al. (2007) Phase II tria of chemoradiation for organ preservation in resectable stage III or IV squamous cell carcinomas of the larynx or oropharynx: results of Eastern Cooperative Oncology Group Study E2399. J Clin Oncol 25: 3971-3977. [Crossref]

27. Aisner J, Sinibaldi V, Eisenberger M (1992) Carboplatin in the treatment of squamous cell head and neck cancers. Semin Oncol 19: 60-65. [Crossref]

28. Coughlin CT, Richmond RC (1989) Biologic and clinical developments of cisplatin combined with radiation: Concepts, utility, projections for new trials, and the emergence of carboplatin. Semin Oncol 16: 31-43. [Crossref]

29. Jeremic B, Shibamoto Y, Stanisavljevic B, Milojevic L, Milicic B, et al. (1997) Radiation therapy alone or with concurrent low-dose daily either cisplatin or carboplatin in locally advanced unresectable squamous cell carcinoma of the head and neck: a prospective randomized trial. Radiother Oncol 43: 29-37. [Crossref]

30. Sanchíz F, Millá A, Torner J, Bonet F, Artola N, et al. (1990) Single fraction per day versus two fractions per day versus radiochemotherapy in the treatment of head and neck cancer. Int J Radiat Oncol Biol Phys 19: 1347-1350. [Crossref]

31. Lo TC, Wiley AL Jr, Ansfield FJ, Brandenburg JH, Davis HL Jr, et al. (1976) Combined radiation therapy and 5-fluorouracil for advanced squamous cell carcinoma of the oral cavity and oropharynx: a randomized study. AJR Am J Roentgenol 126: 229-235. [Crossref]

32. Pergolizzi S, Adamo V, Ferraro G, Sergi C, Santacaterina A, et al. (2004) Induction chemotherapy to weekly paclitaxel concurrent with curative radiotherapy in stage IV (M0) unresectable head and neck squamous cell carcinoma: a dose escalation study. $J$ Chemother 16: 201-205. [Crossref]

33. Agarwala SS, Cano E, Heron DE, Johnson J, Myers E, et al. (2007) Long-term outcomes with concurrent carboplatin, paclitaxel and radiation therapy for locally advanced, inoperable head and neck cancer. Annals of Oncology 18: 1224-1229. [Crossref]

34. Adelstein DJ, Li Y, Adams GL, Wagner H Jr, Kish JA, et al. (2003) An intergroup phase III comparison of standard radiation therapy and two schedules of concurrent chemoradiotherapy in patients with unresectable squamous cell head and neck cancer. J Clin Oncol 21: 92-98. [Crossref]

35. Al-Sarraf M, LeBlanc M, Giri PG, Fu KK, Cooper J, et al. (1998) Chemoradiotherapy versus radiotherapy in patients with advanced nasopharyngeal cancer: phase III randomized intergroup study 0099. J Clin Oncol 16: 1310-1317. [Crossref]

36. Calais G, Alfonsi M, Bardet E, Sire C, Germain T, et al. (1999) Randomized trial of radiation therapy versus concomitant chemotherapy and radiation therapy for advanced-stage oropharynx carcinoma. J Natl Cancer Inst 91: 2081-2086. [Crossref]

37. Forastiere AA, Goepfert H, Maor M, Pajak TF, Weber R, et al. (2003) Concurrent chemotherapy and radiotherapy for organ preservation in advanced laryngeal cancer. N Engl J Med 349: 2091-2098.

38. Pignon JP, Bourhis J, Domenge C, Designe L (2000) Chemotherapy added to locoregional treatment for head and neck squamous cell carcinoma: three metaanalyses of updated individual data. MACH-NC Collaborative Group. Meta-Analysis of Chemotherapy on Head and Neck Cancer. Lancet 355: 949-955. [Crossref]

39. Denaro N, Russi EG, Merlano MC (2013) Strategies for non-resectable head and neck cancer. Curr Treat Options Oncol 14: 492-504. [Crossref]

Copyright: @2018 Shuayb. This is an open-access article distributed under the terms of the Creative Commons Attribution License, which permits unrestricted use, distribution, and reproduction in any medium, provided the original author and source are credited. 